\title{
The Identical Estimates of Spectral Norms for Circulant Matrices with Binomial Coefficients Combined with Fibonacci Numbers and Lucas Numbers Entries
}

\author{
Jianwei Zhou \\ Department of Mathematics, Linyi University, Linyi 276005, China \\ Correspondence should be addressed to Jianwei Zhou; jwzhou@yahoo.com \\ Received 1 November 2013; Accepted 23 January 2014; Published 27 February 2014 \\ Academic Editor: Yuming Xing \\ Copyright (C) 2014 Jianwei Zhou. This is an open access article distributed under the Creative Commons Attribution License, which \\ permits unrestricted use, distribution, and reproduction in any medium, provided the original work is properly cited. \\ Improved estimates for spectral norms of circulant matrices are investigated, and the entries are binomial coefficients combined \\ with either Fibonacci numbers or Lucas numbers. Employing the properties of given circulant matrices, this paper improves the \\ inequalities for their spectral norms, and gets corresponding identities of spectral norms. Moreover, by some well-known identities, \\ the explicit identities for spectral norms are obtained. Some numerical tests are listed to verify the results.
}

\section{Introduction}

Circulant matrices have connection to physics, signal and image processing, probability, statistics, numerical analysis, algebraic coding theory, and many other areas. There are lots of examples from statistical signal processing and information theory that illustrate the application of the circulant matrices, which emphasize how the asymptotic eigenvalue distribution theorem allows one to evaluate results for processes (for the details please refer to [1-3] and the reference therein). Meanwhile a real circulant stochastic process can be described with autocovariance matrices, which are subjected to a cyclical permutation. With the help of autocovariance circulant matrices, it is easy to provide derivations of some results that are central to the analysis of statistical periodograms and empirical spectral density functions (see [4]).

In past decades, the estimates for spectral norms of matrices have been investigated in lots of literatures. Moreover, the determinants and inverses of circulant matrices are stated in many articles. The norms of circulant matrices play an important role in analysing the process of statistics, numerical analysis, and many other problems (for more details, please refer to $[3,5-10]$ and the reference therein). Bryc and Sethuraman [11] investigated the maximum eigenvalue for circulant matrices. Solak [7] obtained lower and upper bounds for the spectral norm of circulant matrices, where the entries are classical Fibonacci numbers. İpek [8] establishes spectral norms of circulant matrices with Fibonacci and Lucas numbers. Furthermore, circulant matrices take up an important status in stochastic calculus, Meckes [12, 13] gave some results on the spectral norm of a special random Toeplitz matrix and random circulant matrices, Mehta [14] made a deep discussion on random circulant matrices.

The outline of this paper is as follows. In Section 2, we state some preliminaries and recall some well-known results. In Section 3, we focus on the identities of estimations for spectral norms. In Section 4, we present various numerical examples to exhibit the accuracy and efficiency of our results. Finally, we summarise this paper and illustrate our future work.

\section{Preliminaries}

The Fibonacci and Lucas sequences $\left\{F_{n}\right\}$ and $\left\{L_{n}\right\}$ are defined by the recurrence relations:

$$
F_{n}=F_{n-1}+F_{n-2}, \quad L_{n}=L_{n-1}+L_{n-2}
$$

with $F_{0}=0, F_{1}=1, L_{0}=2$, and $L_{1}=1$, respectively. 
Obviously, the Fibonacci and Lucas sequences are listed in the following sequence:

$$
\begin{array}{cccccccccc}
n & 0 & 1 & 2 & 3 & 4 & 5 & 6 & 7 & \cdots \\
F_{n} & 0 & 1 & 1 & 2 & 3 & 5 & 8 & 13 & \cdots \\
L_{n} & 2 & 1 & 3 & 4 & 7 & 11 & 18 & 29 & \cdots
\end{array}
$$

and their corresponding Binet forms are (see [15])

$$
\begin{gathered}
F_{n}=\frac{1}{\sqrt{5}}\left[\left(\frac{1+\sqrt{5}}{2}\right)^{n}-\cos (\pi n)\left(\frac{1+\sqrt{5}}{2}\right)^{-n}\right], \\
L_{n}=\left(\frac{1+\sqrt{5}}{2}\right)^{n}+\cos (\pi n)\left(\frac{1+\sqrt{5}}{2}\right)^{-n} .
\end{gathered}
$$

Now, we recall that, for

$$
B=\left(\begin{array}{cccc}
F_{0} & F_{1} & \cdots & F_{n-1} \\
F_{n-1} & F_{0} & \cdots & F_{n-2} \\
F_{n-2} & F_{n-1} & \cdots & F_{n-3} \\
\vdots & \vdots & \ddots & \vdots \\
F_{1} & F_{2} & \cdots & F_{0}
\end{array}\right)_{n \times n}
$$

there hold the following estimates:

$$
\sqrt{F_{n} F_{n-1}+4\left(F_{n-1}^{2}+F_{n-1} F_{n-2}+\frac{1-(-1)^{n}}{2}\right)} \leq\|B\|_{2} \text {, }
$$

$\|B\|_{2}$

$$
\begin{aligned}
& \leq \sqrt{F_{n} F_{n-1}+4\left(F_{n-1}^{2}+F_{n-1} F_{n-2}+\frac{1-(-1)^{n}}{2}\right)} \\
& \quad \times \sqrt{1+F_{n} F_{n-1}+4\left(F_{n-1}^{2}+F_{n-1} F_{n-2}-\frac{3+(-1)^{n}}{2}\right)} .
\end{aligned}
$$

For the details please refer to [7].

There are lots of identities for Fibonacci numbers and Lucas numbers combined with Binomial coefficients (for more details please refer to $[8,16-18]$ and the reference therein). In this paper, we focus on the following identities:

$$
\begin{array}{cc}
\sum_{i=0}^{n}\left(\begin{array}{c}
n \\
i
\end{array}\right) F_{i}=F_{2 n}, \quad \sum_{i=0}^{n}\left(\begin{array}{c}
n \\
i
\end{array}\right) L_{i}=L_{2 n}, \\
\sum_{i=0}^{n}\left(\begin{array}{c}
n \\
i
\end{array}\right) F_{i+k}=F_{2 n+k}, \quad \sum_{i=0}^{n}\left(\begin{array}{c}
n \\
i
\end{array}\right) L_{i+k}=L_{2 n+k} .
\end{array}
$$

Furthermore, for all $k \in \mathbb{Z}^{+}$, there hold the following identities:

$$
\begin{aligned}
& \sum_{i=0}^{n}\left(\begin{array}{c}
n \\
i
\end{array}\right) F_{2 i+k}= \begin{cases}5^{n / 2} F_{n+k}, & (n \text { even }), \\
5^{(n-1) / 2} L_{n+k}, & (n \text { odd }),\end{cases} \\
& \sum_{i=0}^{n}\left(\begin{array}{c}
n \\
i
\end{array}\right) L_{2 i+k}= \begin{cases}5^{n / 2} L_{n+k} & (n \text { even }), \\
5^{(n+1) / 2} F_{n+k} & (n \text { odd }) .\end{cases}
\end{aligned}
$$

Definition 1 (see [19]). A circulant matrix is an $n \times n$ complex matrix with the following form:

$$
A=\left(\begin{array}{cccc}
a_{0} & a_{1} & \cdots & a_{n-1} \\
a_{n-1} & a_{0} & \cdots & a_{n-2} \\
a_{n-2} & a_{n-1} & \cdots & a_{n-3} \\
\vdots & \vdots & \ddots & \vdots \\
a_{1} & a_{2} & \cdots & a_{0}
\end{array}\right)_{n \times n} .
$$

The first row of $A$ is $\left(a_{0}, a_{1}, \ldots, a_{n-1}\right)$ and its $(j+1)$ th row is obtained by giving its $j$ th row a right circular shift by one positions.

Definition 2 (see [3]). The spectral norm $\|\cdot\|_{2}$ of a matrix $A$ with complex entries is the square root of the largest eigenvalue of the positive semidefinite matrix $A^{*} A$ :

$$
\|A\|_{2}=\sqrt{\lambda_{\max }\left(A^{*} A\right)}
$$

where $A^{*}$ denotes the conjugate transpose of $A$. Therefore if $A$ is an $n \times n$ real symmetric matrix or $A$ is a normal matrix, then

$$
\|A\|_{2}=\max _{1 \leq i \leq n}\left|\lambda_{i}\right|
$$

where $\lambda_{1}, \lambda_{2}, \ldots, \lambda_{n}$ are the eigenvalues of $A$.

\section{The Identities of Estimations for Spectral Norms}

We give the main theorems of this paper in the following parts.

Theorem 3. Let $B_{1}$ be as the matrix in (9), and let the first row of $B_{1}$ be $\left(\left(\begin{array}{c}n \\ 0\end{array}\right) F_{0},\left(\begin{array}{c}n \\ 1\end{array}\right) F_{1}, \ldots,\left(\begin{array}{l}n \\ n\end{array}\right) F_{n}\right)$. Then one has

$$
\left\|B_{1}\right\|_{2}=F_{2 n} \text {. }
$$

Proof. Combining with Definition 2, the spectral radius of $B_{1}$ is equal to its spectral norm, where we used the fact that $B_{1}$ is normal. Moreover, by the irreducible and entrywise nonnegative properties, we deduce that $\left\|B_{1}\right\|_{2}$ is equal to its Perron value. Denote by $v=(1,1, \ldots, 1)^{T}$ an $n$-dimensional column vector. There holds

$$
B_{1} v=\left(\sum_{i=0}^{n}\left(\begin{array}{c}
n \\
i
\end{array}\right) F_{i}\right) v .
$$

Obviously, $\sum_{i=0}^{n}\left(\begin{array}{c}n \\ i\end{array}\right) F_{i}$ is an eigenvalue of $B_{1}$ associated with the positive eigenvector $v$, which is the Perron value of $B_{1}$. Employing the first identity in (6), we have

$$
\left\|B_{1}\right\|_{2}=F_{2 n} \text {. }
$$

This completes the proof. lary.
With the same approach, we obtain the following corol- 
TABLE 1: Spectral norms of $B_{i}(i=1,2,3,4)$ and $k=1$.

\begin{tabular}{lllllllcc}
\hline$n$ & 1 & 2 & 3 & 4 & 5 & 6 & 7 \\
$\left\|B_{1}\right\|_{2}$ & 1 & 3 & 8 & 21 & 55 & 144 & 377 & 377 \\
$F_{2 n}$ & 1 & 3 & 8 & 21 & 55 & 144 & 987 \\
$\left\|B_{2}\right\|_{2}$ & 3 & 7 & 18 & 47 & 123 & 322 & 2207 \\
$L_{2 n}$ & 3 & 7 & 18 & 47 & 123 & 322 & 843 \\
$\left\|B_{3}\right\|_{2}$ & 2 & 5 & 13 & 34 & 89 & 233 & 610 \\
$F_{2 n+1}$ & 2 & 5 & 13 & 34 & 89 & 233 & 610 \\
$\left\|B_{4}\right\|_{2}$ & 4 & 11 & 29 & 76 & 199 & 521 & 1364 \\
$L_{2 n+1}$ & 4 & 11 & 29 & 76 & 199 & 521 & 1397 \\
\hline
\end{tabular}

Corollary 4. Let $B_{2}$ be as the matrix in (9), and let the first row of $B_{2}$ be $\left(\left(\begin{array}{c}n \\ 0\end{array}\right) L_{0},\left(\begin{array}{l}n \\ 1\end{array}\right) L_{1}, \ldots,\left(\begin{array}{l}n \\ n\end{array}\right) L_{n}\right)$. Then one has the following identity:

$$
\left\|B_{2}\right\|_{2}=L_{2 n}
$$

Theorem 5. Let $B_{3}$ be with the form as (9). For all $k \in \mathbb{Z}^{+}$, if the first row of $B_{3}$ is $\left(\left(\begin{array}{c}n \\ 0\end{array}\right) F_{k},\left(\begin{array}{c}n \\ 1\end{array}\right) F_{1+k}, \ldots,\left(\begin{array}{l}n \\ n\end{array}\right) F_{n+k}\right)$, then one obtains

$$
\left\|B_{3}\right\|_{2}=F_{2 n+k} \text {. }
$$

Proof. Following the same techniques of the above theorem and combining with the fact that $B_{3}$ is irreducible and entrywise nonnegative, we declare that the spectral norm of $B_{3}$ is equal to its Perron value. Let $v^{T}=(1,1, \ldots, 1)_{1 \times n}$. Then

$$
B_{3} v=\left(\sum_{i=0}^{n}\left(\begin{array}{l}
n \\
i
\end{array}\right) F_{i+k}\right) v .
$$

Obviously, we declare that $\sum_{i=0}^{n}\left(\begin{array}{c}n \\ i\end{array}\right) F_{i+k}$ is an eigenvalue of $B_{3}$ associated with $v$. With simple analysis, we obtain that $\sum_{i=0}^{n}\left(\begin{array}{c}n \\ i\end{array}\right) F_{i+k}$ is equal to the Perron value of $B_{3}$. Combining with the third identity of binomial coefficients and Fibonacci numbers in (6), we obtain

$$
\left\|B_{3}\right\|_{2}=F_{2 n+k}
$$

which completes the proof.

Similarly, there holds the following corollary.

Corollary 6. Let $B_{4}$ be as the matrix in (9). For all $k \in \mathbb{Z}^{+}$, the first row of $B_{4}$ is $\left(\left(\begin{array}{c}n \\ 0\end{array}\right) L_{k},\left(\begin{array}{l}n \\ 1\end{array}\right) L_{1+k}, \ldots,\left(\begin{array}{l}n \\ n\end{array}\right) L_{n+k}\right)$; then

$$
\left\|B_{4}\right\|_{2}=L_{2 n+k} \text {. }
$$

Now, we are at the point to recall the following lemma to verify the identities of spectral norms with other approaches.

Lemma 7 (see [3]). Let $A$ be a nonnegative matrix. If the column sums of $A$ are equal, then

$$
\rho(A)=\|A\|_{1},
$$

where $\rho(A)=\max \{|\lambda|: \lambda$ is an eigenvalue of matrix $A\}$ and $\|\cdot\|_{1}$ denotes the maximum column sum matrix norm.
Theorem 8. Let $B_{5}$ be with the form as (9) and let the first row of $B_{5}$ be $\left(\left(\begin{array}{c}n \\ 0\end{array}\right) F_{k},\left(\begin{array}{c}n \\ 1\end{array}\right) F_{2+k}, \ldots,\left(\begin{array}{c}n \\ n\end{array}\right) F_{2 n+k}\right)$. Then one deduces the following identity:

$$
\left\|B_{5}\right\|_{2}= \begin{cases}5^{n / 2} F_{n+k} & (n \text { even }), \\ 5^{(n-1) / 2} L_{n+k} & (n \text { odd }),\end{cases}
$$

where $k \in \mathbb{Z}^{+}$.

Proof. Obviously, the circulant matrix $B_{5}$ is normal; with the results of Definition 2, we declare that the spectral radius of $B_{5}$ is equal to $\rho\left(B_{5}\right)$; that is, $\left\|B_{5}\right\|_{2}=\rho\left(B_{5}\right)$. Furthermore, applying entrywise nonnegative properties and column sum of $B_{5}$ are certain constant $K_{\text {col }}$, which is described in (7). By Lemma 7, we obtain

$$
\rho\left(B_{5}\right)=K_{\mathrm{col}} \cdot
$$

Employing the identities of Fibonacci numbers and Binomial coefficients in (7), we have

$$
\left\|B_{5}\right\|_{2}= \begin{cases}5^{n / 2} F_{n+k} & (n \text { even }), \\ 5^{(n-1) / 2} L_{n+k} & (n \text { odd }) .\end{cases}
$$

This completes the proof.

Furthermore, we give the following corollary without proofs, which can be proved with the same approaches as the above theorem.

Corollary 9. Let $B_{6}$ be as the matrix in (9). For all $k \in \mathbb{Z}^{+}$, the first row of $B_{6}$ is $\left(\left(\begin{array}{l}n \\ 0\end{array}\right) L_{k},\left(\begin{array}{l}n \\ 1\end{array}\right) L_{2+k}, \ldots,\left(\begin{array}{l}n \\ n\end{array}\right) L_{2 n+k}\right)$; then we have the following identity:

$$
\left\|B_{6}\right\|_{2}= \begin{cases}5^{n / 2} L_{n+k} & (\text { n even }), \\ 5^{(n+1) / 2} F_{n+k} & (n \text { odd }) .\end{cases}
$$

\section{Numerical Examples}

In this section, we give some examples to verify our identities in the above theorems and corollaries.

Example 10. In this example, we give the numerical results for $B_{i}(i=1,2,3,4)$ in Table 1 . 
TABLE 2: Spectral norms of $B_{6}$ and $B_{6}$.

\begin{tabular}{lcccccccc}
\hline$n$ & 1 & 2 & 3 & 4 & 5 & 6 & 7 \\
$\left\|B_{5}\right\|_{2}$ & 3 & 10 & 35 & 125 & 450 & 1625 & 5875 \\
$5^{n / 2} F_{n+1}$ & $\backslash$ & 10 & $\backslash$ & 125 & $\backslash$ & 1625 & 21250 \\
$5^{(n-1) / 2} L_{n+1}$ & 3 & $\backslash$ & 35 & $\backslash$ & 450 & $\backslash$ & 21250 \\
$\left\|B_{6}\right\|_{2}$ & 5 & 20 & 100 & 275 & 1000 & 3625 & 13125 \\
$5^{(n+1) / 2} F_{n+1}$ & 5 & $\backslash$ & 100 & $\backslash$ & 1000 & $\backslash$ & 13125 \\
$5^{n / 2} L_{n+1}$ & $\backslash$ & 20 & $\backslash$ & 275 & $\backslash$ & 47500 \\
\hline
\end{tabular}

Example 11. For simplicity, let $k=1$. We give the numerical results for $B_{5}$ and $B_{6}$ in Table 2 .

With the data in Tables 1 and 2, we declare that the identity for the spectral norm of $B_{i}(i=1, \ldots, 6)$ holds.

\section{Conclusion}

This paper had discussed the identical estimates of spectral norms for some circulant matrices, which are listed by explicit formulations. In the future, we are going to investigate the determinants, inverses of circulant matrices with certain entries, and, inspired by [6], we will investigate the properties of $g$-circulant matrices. Particularly worth mentioning is the fact that, for the $g$-circulant matrix, we had some numerical results to prove the fact that the same identical estimates hold precisely, and we will concern on the theoretical confirmation in part of the future work.

\section{Conflict of Interests}

The author declares that there is no conflict of interests regarding the publication of this paper.

\section{Acknowledgments}

The author thanks Professor Z. L. Jiang for valuable discussions and suggestions and wishes to express sincere thanks to referees for their useful suggestions and comments. This work is partly supported by National Natural Science Foundation of China (Grant no. 11201212), Promotive Research Fund for Excellent Young and Middle-Aged Scientists of Shandong Province (Grant no. BS2012DX004), and the AMEP of Linyi University.

\section{References}

[1] W.-S. Chou, B.-S. Du, and P. J.-S. Shiue, "A note on circulant transition matrices in Markov chains," Linear Algebra and Its Applications, vol. 429, no. 7, pp. 1699-1704, 2008.

[2] R. M. Gray and L. D. Davisson, An Introduction to Statistical Signal Processing, Cambridge University Press, London, UK, 2005.

[3] R. A. Horn and C. R. Johnson, Matrix Analysis, Cambridge University Press, Cambridge, UK, 1985.
[4] D. S. G. Pollock, "Circulant matrices and time-series analysis," Working Paper No. 442, Queen Mary \& Westfield College, 2000.

[5] A. Bose, R. S. Hazra, and K. Saha, "Spectral norm of circulanttype matrices," Journal of Theoretical Probability, vol. 24, no. 2, pp. 479-516, 2011.

[6] E. Ngondiep, S. Serra-Capizzano, and D. Sesana, "Spectral features and asymptotic properties for $g$-circulants and $g$ Toeplitz sequences," SIAM Journal on Matrix Analysis and Applications, vol. 31, no. 4, pp. 1663-1687, 2010.

[7] S. Solak, "On the norms of circulant matrices with the Fibonacci and Lucas numbers," Applied Mathematics and Computation, vol. 160, no. 1, pp. 125-132, 2005.

[8] A. İpek, "On the spectral norms of circulant matrices with classical Fibonacci and Lucas numbers entries," Applied Mathematics and Computation, vol. 217, no. 12, pp. 6011-6012, 2011.

[9] J. W. Zhou and Z. L. Jiang, "Spectral norms of circulant-type matri-ces with Binomial coefficients and Harmonic numbers," International Journal of Computational Methods, vol. 11, no. 5, Article ID 1350076, 14 pages, 2014.

[10] J. W. Zhou and Z. L. Jiang, "Spectral norms of circulant and Skew-circulant matrices with Binomial coefficients entries," in Proceedings of the 9th International Symposium on Linear Drives for Industry Applications, vol. 271 of Lecture Notes in Electrical Engineering, pp. 219-224, Springer, Berlin, Germany, 2014.

[11] W. Bryc and S. Sethuraman, "A remark on the maximum eigenvalue for circulant matrices," in High Dimensional Probability V: The Luminy Volume, vol. 5, pp. 179-184, Institute of Mathematical Statistics Collections, Beachwood, Ohio, USA, 2009.

[12] M. W. Meckes, "On the spectral norm of a random Toeplitz matrix," Electronic Communications in Probability, vol. 12, pp. 315-325, 2007.

[13] M. W. Meckes, "Some results on random circulant matrices," in High Dimensional Probability V: The Luminy Volume, vol. 5, pp. 213-223, Institute of Mathematical Statistics Collections, Beachwood, Ohio, USA, 2009.

[14] M. L. Mehta, Random Matrices, vol. 142 of Pure and Applied Mathematics, Elsevier/Academic Press, Amsterdam, The Netherlands, 3rd edition, 2004.

[15] E. G. Kocer, N. Tuglu, and A. Stakhov, "On the $m$-extension of the Fibonacci and Lucas p-numbers," Chaos, Solitons and Fractals, vol. 40, no. 4, pp. 1890-1906, 2009.

[16] M. Akbulak and D. Bozkurt, "On the norms of Toeplitz matrices involving Fibonacci and Lucas numbers," Hacettepe Journal of Mathematics and Statistics, vol. 37, no. 2, pp. 89-95, 2008. 
[17] M. Benoumhani, "A sequence of Binomial coefficients related to Lucas and Fibonacci numbers," Journal of Integer Sequences, vol. 6, no. 2, pp. 1-10, 2003.

[18] R. Melham, "Sums involving Fibonacci and Pell numbers," Portugaliae Mathematica, vol. 56, no. 3, pp. 309-317, 1999.

[19] W. T. Stallings and T. L. Boullion, "The pseudoinverse of an $r$-circulant matrix," Proceedings of the American Mathematical Society, vol. 34, no. 2, pp. 385-388, 1972. 


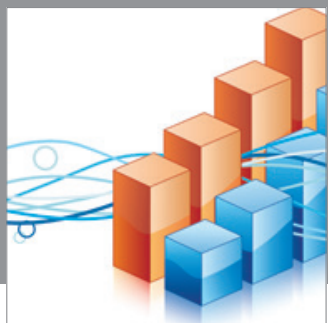

Advances in

Operations Research

mansans

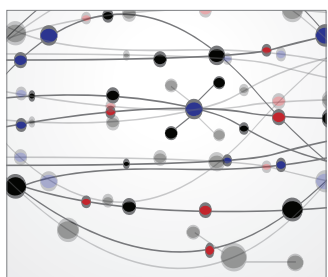

The Scientific World Journal
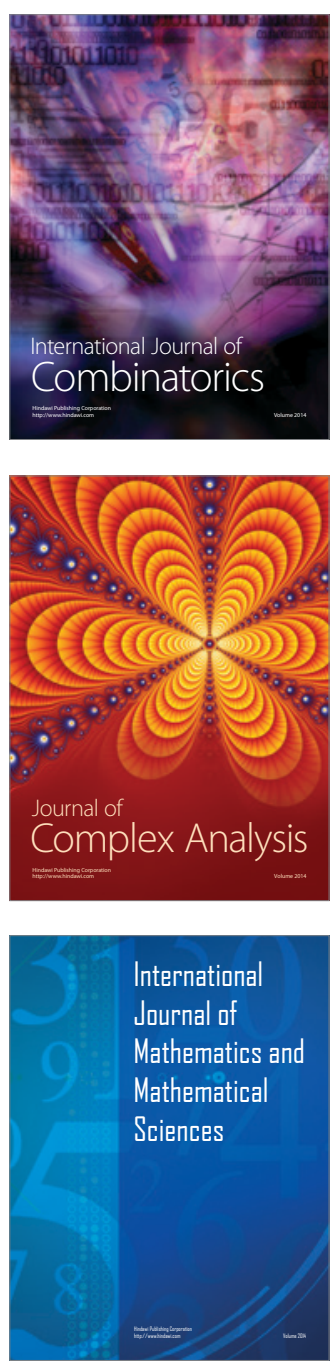
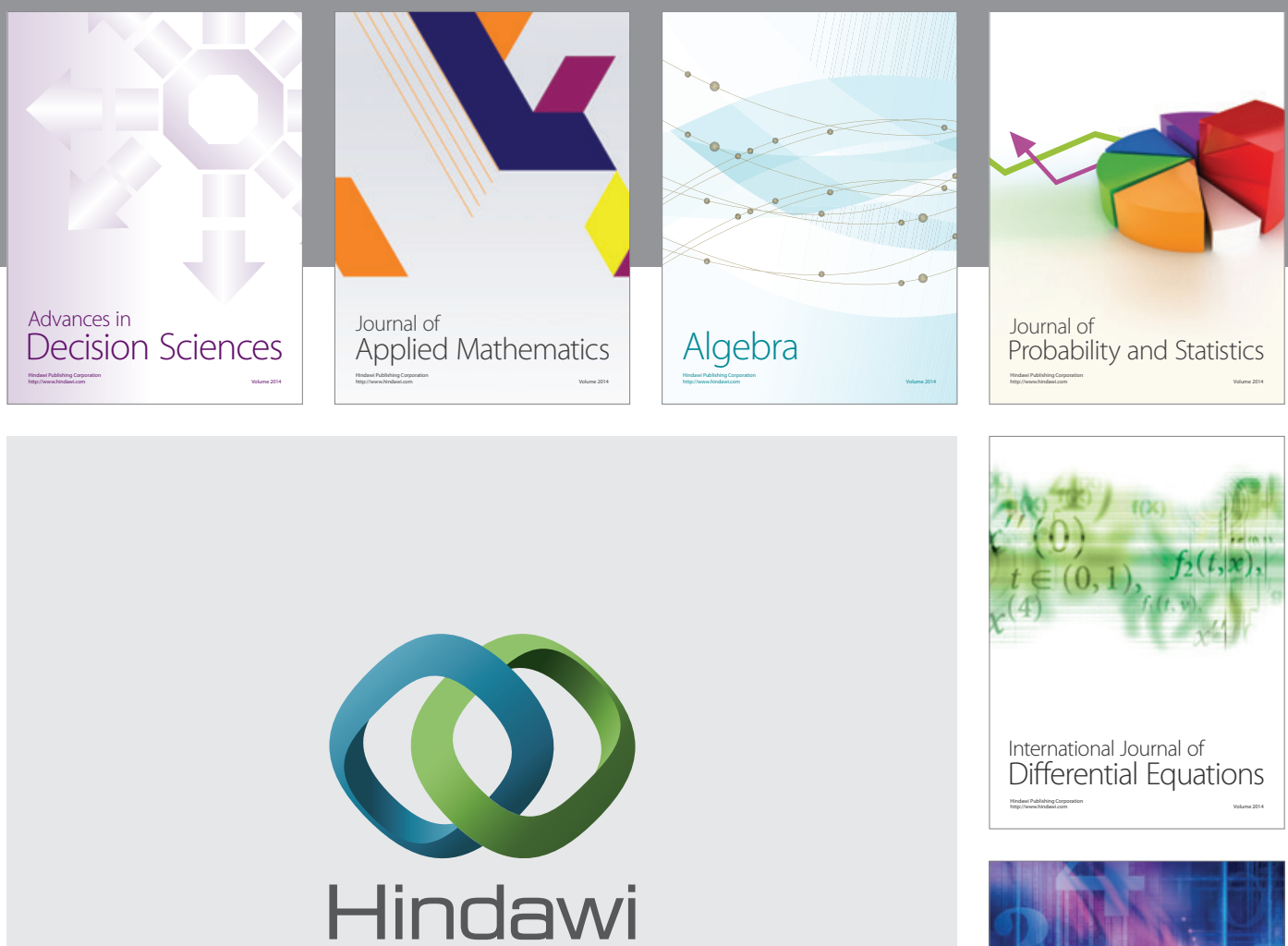

Submit your manuscripts at http://www.hindawi.com
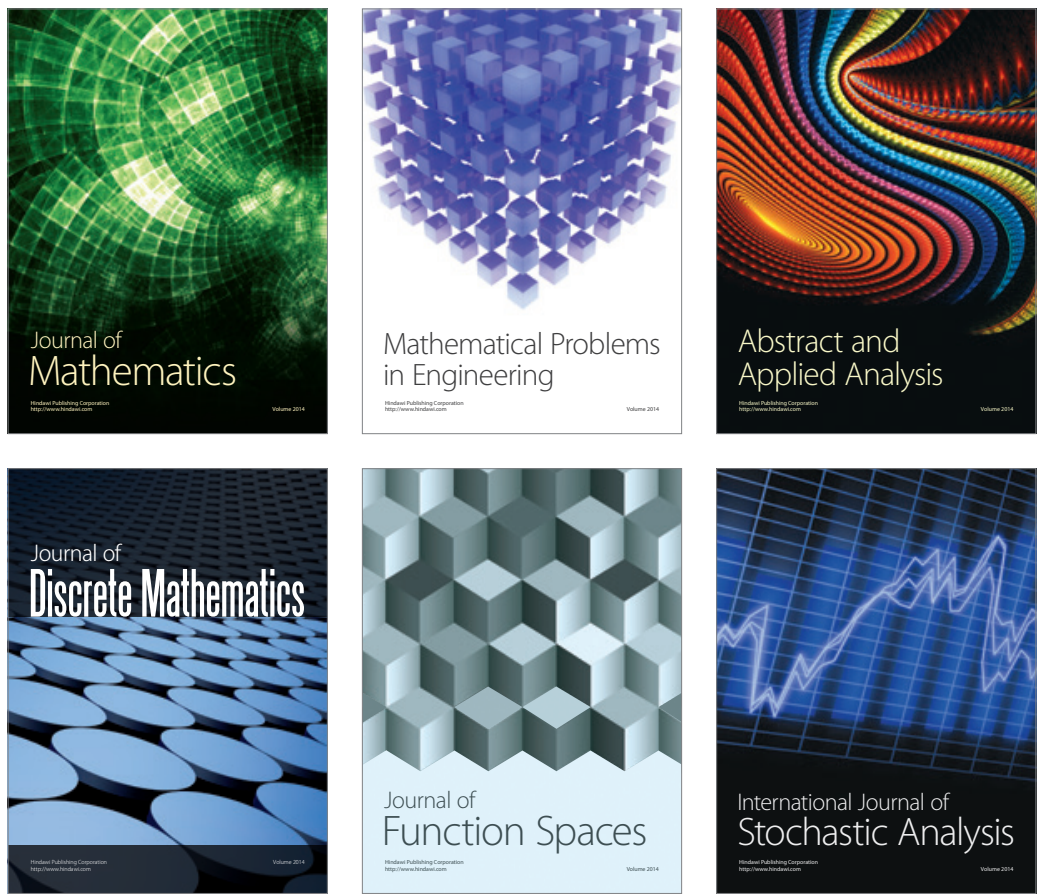

Journal of

Function Spaces

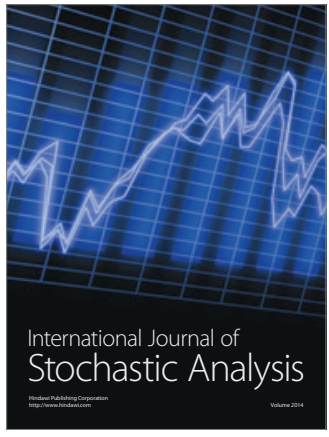

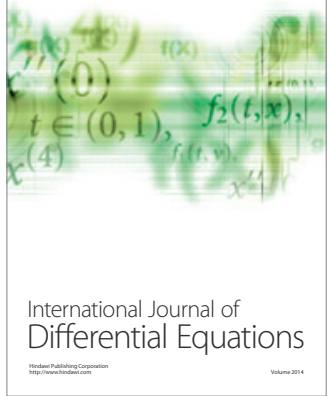
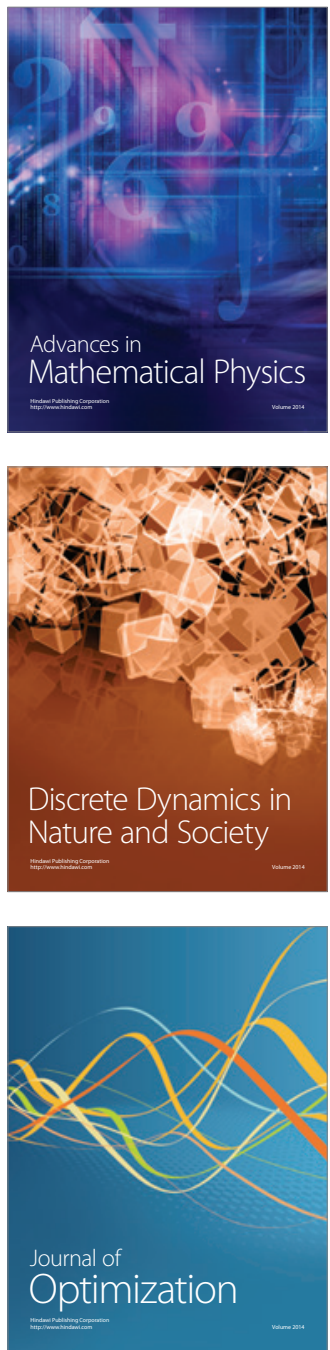